\title{
MAXIMAL IDEALS IN SUBALGEBRAS OF $C(X)$
}

\author{
LOTHAR REDLIN AND SALEEM WATSON
}

\begin{abstract}
Let $X$ be a completely regular space, and let $A(X)$ be a subalgebra of $C(X)$ containing $C^{*}(X)$. We study the maximal ideals in $A(X)$ by associating a filter $Z(f)$ to each $f \in A(X)$. This association extends to a oneto-one correspondence between $\mathcal{M}(A)$ (the set of maximal ideals of $A(X)$ ) and $\beta X$. We use the filters $Z(f)$ to characterize the maximal ideals and to describe the intersection of the free maximal ideals in $A(X)$. Finally, we outline some of the applications of our results to compactifications between $v X$ and $\beta X$.
\end{abstract}

1. Introduction. The algebra $C(X)$ of continuous real-valued functions on a completely regular space $X$ and its subalgebra $C^{*}(X)$ of bounded functions have been studied extensively (see Gillman and Jerison [3], and Aull [1]). One of the interesting problems considered in [3] is that of characterizing the maximal ideals in these two algebras. It is a remarkable fact that the distinct problems of identifying the maximal ideals in $C(X)$ and $C^{*}(X)$ have a common solution-the maximal ideals are in one-to-one correspondence with the points of $\beta X$ in a natural way. The methods of achieving this correspondence, however, are quite different in the two cases. In this paper we consider this problem for subalgebras $A(X)$ of $C(X)$ that contain $C^{*}(X)$. We show that for such algebras the maximal ideals are in one-to-one correspondence with $\beta X$. The correspondence we construct reduces to that in [3] for the cases of $C(X)$ and $C^{*}(X)$. Thus our result puts in a common setting these apparently distinct problems.

A function is invertible in $C(X)$ if it is never zero, and in $C^{*}(X)$ if it is bounded away from zero. In an arbitrary $A(X)$, of course, there is no such description of invertibility which is independent of the structure of the algebra. Thus in $\S 2$ we associate to each noninvertible $f \in A(X)$ a $z$-filter $Z(f)$ that is a measure of where $f$ is "locally" invertible in $A(X)$. This correspondence extends to one between maximal ideals of $A(X)$ and $z$-ultrafilters on $X$. In $\S 3$ we use the filters $Z(f)$ to describe the intersection of the free maximal ideals in any algebra $A(X)$. Finally, our main result allows us to introduce the notion of $A(X)$-compactness of which compactness and realcompactness are special cases. In $\S 4$ we show how the Banach-Stone theorem extends to $A(X)$-compact spaces.

2. The structure space. Throughout this paper $X$ will denote a completely regular Hausdorff space and $A(X)$ a subalgebra of $C(X)$ containing $C^{*}(X)$. In this section we construct the correspondence mentioned in the introduction.

A zero set in $X$ is a set of the form $Z(f)=\{x \in X: f(x)=0\}$ for some $f \in C(X)$. The complement of a zero set is a cozero set. $Z[X]$ will denote the

Received by the editors April 18, 1986.

1980 Mathematics Subject Classification (1985 Revision). Primary 54C40; Secondary 46E25.

Key words and phrases. Algebras of continuous functions, maximal ideal, compactifications. 
collection of all zero sets in $X$. If $E$ is a cozero set in $X$ we will say that $f \in A(X)$ is $E$-regular if there exists $g \in A(X)$ such that $\left.f g\right|_{E}=1$.

LEMMA 1. Let $f, g \in A(X)$ and let $E, F$ be cozero sets in $X$.

(a) If $f$ is $E$-regular and $F \subseteq E$, then $f$ is $F$-regular.

(b) If $f$ is E-regular and $F$-regular, then $f$ is $E \cup F$-regular.

(c) If $f(x) \geq c>0$ for all $x \in E$, then $f$ is E-regular.

(d) If $0<f(x) \leq g(x)$ for all $x \in E$ and if $f$ is E-regular, then $g$ is E-regular.

(e) If $f$ is E-regular and $g$ is $F$-regular, then $f g$ is $E \cap F$-regular and $f^{2}+g^{2}$ is $E \cup F$-regular.

ProOF. (a) Obvious.

(b) Let $h, k \in A(X)$ satisfy $\left.h f\right|_{E}=1$ and $\left.k f\right|_{F}=1$. Let $w=h+k-f h k$. Then $\left.f w\right|_{E \cup F}=1$.

(c) Let $h=\max \{c, f\}$. Then $\left.h\right|_{E}=\left.f\right|_{E}$ and $h \geq c$. So $0<h^{-1} \leq c^{-1}$. Hence $h^{-1} \in C^{*}(X) \subseteq A(X)$, and $\left.h^{-1} f\right|_{E}=1$.

(d) Let $h \in A(X)$ satisfy $\left.h f\right|_{E}=1$. For $x \in E, h(x)>0$, so $h(x) g(x) \geq$ $h(x) f(x)=1$. Thus by (c), there exists $k \in A(X)$ such that $\left.k h g\right|_{E}=1$.

(e) If $\left.h f\right|_{E}=1$ and $\left.k g\right|_{F}=1$, then $\left.h k g f\right|_{E \cap F}=1$. Now $f^{2}+g^{2} \geq f^{2}$, so by (d), $f^{2}+g^{2}$ is $E$-regular. Similarly, it is $F$-regular, and so the result follows by (b).

For $f \in A(X)$, we define

$$
Z(f)=\left\{E \in Z[X]: f \text { is } E^{c} \text {-regular }\right\},
$$

and for $S \subseteq A(X), Z[S]=\bigcup_{f \in S} Z(f)$. We recall that a $z$-filter is a nonempty collection $\mathcal{F}$ of zero sets in $X$ such that $\mathcal{F}=\mathcal{G} \cap Z[X]$, for some filter $\mathcal{G}$ on $X$.

THEOREM 1. If $f$ is not invertible in $A(X)$, then $Z(f)$ is a $z$-filter on $X$, and conversely.

Proof. If $f$ is not invertible, $\varnothing \notin Z(f)$. Moreover, if $E, F \in Z(f)$, then by Lemma 1 (b), $E \cap F \in Z(f)$. If $G$ is a zero set containing $E \in Z(f)$, then $G \in Z(f)$ by Lemma $1(\mathrm{a})$. Hence $Z(f)$ is a $z$-filter.

The converse is obvious.

THEOREM 2. If $I$ is an ideal in $A(X)$, then $Z[I]$ is a $z$-filter on $X$.

Proof. Clearly $\varnothing \notin Z[I]$. If $E, F \in Z[I]$, there exist $f, g \in I$ such that $f$ is $E^{c}$-regular and $g$ is $F^{c}$-regular. Then $f^{2}+g^{2} \in I$, and by Lemma $1(\mathrm{e}), f^{2}+g^{2}$ is $(E \cap F)^{c}$-regular. Thus $E \cap F \in Z[I]$. Finally, if $F$ is a zero set and $F \supseteq E \in Z[I]$, then $E \in Z(f)$ for some $f \in I$, and so $F \in Z(f) \subseteq Z[I]$ by Theorem 1 .

Using the notation of $[\mathbf{3}]$, we write $Z^{\leftarrow}[\mathcal{F}]=\{f \in A(X): Z(f) \subseteq \mathcal{F}\}$ for the inverse of the set function $Z$. We will show that if $\mathcal{F}$ is a $z$-filter, then $Z^{\leftarrow}[\mathcal{F}]$ is an ideal in $A(X)$, giving a converse to the above theorem. We need two preliminary lemmas.

LEMMA 2. If $f \in A(X)$, then $\lim _{z(f)} f h=0$ for any $h \in A(X)$.

ProOF. We claim $\lim _{z(f)} f=0$. The result will follow from this claim and Lemma $1(\mathrm{e})$, since then $\lim _{Z(f h)} f h=0$ and $Z(f h) \subseteq Z(f)$. So let $V=(-\varepsilon, \varepsilon)$ be a neighborhood of zero in $\mathbf{R}$ and let $E=f^{-1}(V)$. Clearly $f$ is $E^{c}$-regular (Lemma 1 (b) and (c)). Thus $f^{-1}(V) \in Z(f)$ and so $f h$ converges to zero on $Z(f)$. 
LEMMA 3. Let $\mathcal{\exists}$ be a $z$-filter on $X$. If $\lim _{\mathcal{F}} f h=0$ for all $h \in A(X)$, then $Z(f) \subseteq \mathcal{F}$.

Proof. For $E \in Z(f)$ we show that there is an $F \in \mathcal{F}$ such that $F \subseteq E$. Suppose not. Then $F \cap E^{c} \neq \varnothing$ for every $F \in \mathcal{F}$. Let $h \in A(X)$ satisfy $\left.f h\right|_{E^{c}}=1$. It follows that 1 is a cluster point of $\{f h(F): F \in \mathcal{F}\}$, contradicting our hypothesis.

THEOREM 3. For any $z$-filter $\mathcal{F}$ on $X, I=Z^{\leftarrow}[\mathcal{F}]$ is an ideal in $A(X)$.

ProOF. If $f \in I$ and $g \in A(X)$, then $Z(f g) \subseteq Z(f)$ (Lemma $1(\mathrm{e})$ ), so $f g \in I$. Now if $f, g \in I$, then by Lemma $2, \lim _{\mathcal{F}} f h=\lim _{\mathcal{F}} g h=0$ for every $h \in A(X)$. So $\lim _{\mathcal{F}} f h+\lim _{\mathcal{F}} g h=\lim _{\mathcal{F}}(f+g) h=0$ for all $h \in A(X)$, and hence by Lemma $3, Z(f+g) \subseteq \mathcal{F}$. Finally, we note that since $\varnothing \notin \mathcal{F}, I$ consists of noninvertible elements only.

Both $Z$ and $Z^{\leftarrow}$ preserve inclusion and so they map maximal elements to maximal elements. Hence $Z$ provides a one-to-one correspondence between $\beta X$ and the set $\mathcal{M}(A)$ of maximal ideals of $A(X)$. If $\mathcal{M}(A)$ is equipped with the hull-kernel topology, then as in [3] in the cases of $C^{*}(X)$ and $C(X)$, we have the following theorem (see [6] for a different method of arriving at this result).

THEOREM 4. The maximal ideal space $\mathcal{M}(A)$ of $A(X)$ equipped with the hullkernel topology is homeomorphic to $\beta X$.

3. Free maximal ideals. Let $M^{p}$ be the maximal ideal corresponding to $p \in \beta X$ and $U^{p}$ the $z$-ultrafilter on $X$ that converges to $p$, so that $Z\left(M^{p}\right)=U^{p}$. Using our filter $Z(f)$ we see immediately that for $f \in A(X), f \in M^{p}$ if and only if $Z(f) \subseteq \mathcal{U}^{p}$. Thus we have the following analogue of the Gelfand-Kolmogoroff theorem [3, Theorem 7.3] for an arbitrary $A(X)$.

THEOREM 5. For the maximal ideals in $A(X)$, we have

$$
M^{p}=\{f \in A(X): p \text { is a cluster point of } Z(f) \text { in } \beta X\} .
$$

We now describe the intersection of all the free maximal ideals in $A(X)$. An ideal $I$ is free if $\bigcap Z[I]=\varnothing$, otherwise it is fixed. Note that a maximal ideal is free if and only if it is of the form $M^{p}$ for some $p \in \beta X \backslash X$. We call a set $E \subseteq X$ small if every zero set contained in $E$ is compact. Let $\mathcal{K}=\left\{E \in Z[X]: E^{c}\right.$ is small $\}$, and let $A_{K}(X)=\{f \in A(X): Z(f) \subseteq \mathcal{K}\}$.

THEOREM 6. $A_{K}(X)=\bigcap\left\{M^{p}: p \in \beta X \backslash X\right\}$.

ProOF. Let $f \in A_{K}(X)$. If $\mathcal{U}$ is any $z$-ultrafilter on $X$ such that $Z(f) \nsubseteq \mathcal{U}$, then there exist disjoint zero sets $E \in Z(f)$ and $F \in U$. But then $F \subseteq E^{c}$, so $F$ is compact and $\mathcal{U}$ is fixed. It follows that $Z(f)$ is contained in every free $z$ ultrafilter, and so $f$ belongs to every free maximal ideal. Conversely, if $f$ is in every free maximal ideal, then $Z(f)$ belongs to every free $z$-ultrafilter. Suppose $E \in Z(f)$ is not in $\mathcal{K}$. Then $E^{c}$ must contain a noncompact zero set $F$. Since $E \cup F \supseteq E \in Z(f), E \cup F$ belongs to every free $z$-ultrafilter, and hence $F$ belongs to no free $z$-ultrafilter. But clearly every noncompact zero set must belong to some free $z$-ultrafilter. Thus $E$ is in $\mathcal{K}$ and $f \in A_{K}(X)$.

We note that if $X$ is realcompact and $A(X)=C(X)$, then $A_{K}(X)$ is the family of functions on $X$ of compact support and Theorem 8.19 of [3] follows from our Theorem 6. If $A(X)=C^{*}(X)$, then $A_{K}(X)$ is the family of functions on $X$ that vanish at infinity and Lemma 3.2 in [4] is a special case of Theorem 6. 
4. A-compactness. It is well known that $C^{*}$ distinguishes among compact spaces (the Banach-Stone theorem) and that $C$ distinguishes among realcompact spaces (Hewitt's isomorphism theorem). Theorem 4 allows us to define the notion of $A$-compactness which will enable us to place both of these theorems in a common setting (Theorem 7).

A maximal ideal $M$ in $A(X)$ is real if $A(X) / M$ is isomorphic to $\mathbf{R}$. Every fixed maximal ideal is real. If every real maximal ideal is fixed, we will say that $X$ is $A(X)$-compact (or simply $A$-compact). With this definition, a compact space is one that is $C^{*}$-compact while a realcompact space is $C$-compact.

THEOREM 7. Let $X$ be A-compact and $Y$ be $B$-compact. If $A(X)$ is isomorphic to $B(Y)$, then $X$ is homeomorphic to $Y$.

ProOF. Since $X$ is $A$-compact its points correspond to the real maximal ideals of $A(X)$ under the homeomorphism described in Theorem 4. Thus we can recover $X$ from the ring structure of $A(X)$. Since this can be done in the same way for $Y$, the result follows.

Although the converse of the above theorem is trivial if $A$ and $B$ are $C$ or $C^{*}$, in this more general setting the converse is not even true. For a given $X$ there can exist nonisomorphic algebras $A(X)$ and $B(X)$ for which $X$ is both $A$-compact and $B$-compact. For example, let $H(\mathbf{N})$ be the algebra of sequences which occur as the coefficients of the Taylor series representation of functions holomorphic on the open unit disc. Then $\mathbf{N}$ is both $H$-compact (see [2]) and $C$-compact, but $H(\mathrm{~N})$ is obviously not isomorphic to $C(\mathrm{~N})$. Indeed, it is clear from the definition that if $X$ is $A$-compact and $B(X) \supseteq A(X)$, then $X$ is $B$-compact. This raises the question: Does there exist in some sense a "minimal" algebra $A_{m}(X)$ for which $X$ is $A_{m}$-compact, at least up to isomorphism?

We conclude by noting that another characterization of $A$-compactness follows from Mandelker [5]. We call a family $S$ of closed sets in $X A$-stable if every $f \in A(X)$ is bounded on some member of $S$. Then one can show (as in [5]) that a space is $A$-compact if and only if every $A$-stable family of closed sets with the finite intersection property has nonempty intersection.

\section{REFERENCES}

1. C. E. Aull, Rings of continuous functions, Dekker, New York, 1985.

2. R. M. Brooks, A ring of analytic functions, Studia Math. 24 (1964), 191-210.

3. L. Gillman and M. Jerison, Rings of continuous functions, Van Nostrand, Princeton, N. J., 1960.

4. C. W. Kohls, Ideals in rings of continuous functions, Fund. Math. 45 (1957), 28-50.

5. M. Mandelker, Supports of continuous functions, Trans. Amer. Math. Soc. 156 (1971), 73-83.

6. D. Plank, On a class of subalgebras of $C(X)$ with applications to $\beta X \backslash X$, Fund. Math. 64 (1969), 41-54.

Pennsylvania State University, Ogontz Campus, Abington, Pennsylvania 19001

Pennsylvania State University, Delaware County Campus, Media, PennsylVANIA 19063 